\title{
Equilibrium Optimizer-Based Robust Sliding Mode Control of Magnetic Levitation System
}

\author{
Omar Y. Ismael ${ }^{*}$, Mohammed Qasim, Mohanad N. Noaman \\ Department of Systems and Control Engineering, Ninevah University, Nineveh 41001, Iraq
}

Corresponding Author Email: omar.ismael@uoninevah.edu.iq

\section{https://doi.org/10.18280/jesa.540115}

Received: 29 October 2020

Accepted: 6 February 2021

\section{Keywords:}

sliding mode control, robust control, equilibrium optimizer, magnetic levitation system

\begin{abstract}
Magnetic Levitation System (MLS) objective is to levitate objects to the desired height without any contact. MLS is highly nonlinear and inherently unstable. Such a system imposes a challenge when designing robust and high-performance controllers. This paper presents the design of a Sliding Mode (SM) controller with an Integral term called SM-I controller to achieve the desired levitation against nonlinearities and uncertainties of the system. The controller parameters are tuned using the Equilibrium Optimizer (EO) algorithm. The Effectiveness of the proposed controller is validated by simulation results. Simulations are performed for servo tracking with and without perturbations in the MLS parameters. The proposed controller is compared with the conventional SM, LQR, and PID controllers to show its superiority. The results prove that the SM-I is more efficient than the other controllers.
\end{abstract}

\section{INTRODUCTION}

MLS has a practical significance in many engineering areas such as high-speed maglev trains, levitation of wind tunnel models, frictionless bearings, vibration isolation of sensitive machinery, suspension, and manipulation [1-6]. The widespread use of MLS is because it eliminates mechanical contact between moving and stationary parts. Consequently, MLS reduces the friction problem and isolates vibrations. Hence, MLS offers several advantages such as working in a high vacuum environment, reduction in noise, and high accuracy positioning system [7]. MLS uses the principle of electromagnetism to levitate ferromagnetic objects at a certain position within air-space [8].

MLS is considered as a nonlinear system that has an unstable equilibrium point [9] with a very small degree of natural damping and fast dynamics. Therefore, controlling such systems is a strenuous problem [10]. MLS has witnessed a dramatic increase in the amount of research in the last two decades and has been considered as a valuable testbed for control techniques.

A variety of control strategies have been proposed and implemented to control the MLS. A digital fractional-order PID controller is designed [11] and implemented. Self-tuning robust integral of signum of error (RISE) based controller is discussed by Bidikli and Bayrak [8]. To obtain a smoother control signal, the authors used the tanh instead of signum function. Four novel fractional-order sliding mode controllers are presented [12] and experimental results are provided. A novel neural network-based controller is presented by Hayat et al. [13] and a model that considers the angular position of the ball is derived. The neural network is used to estimate the electromagnetic parameters. A sliding mode controller is designed to reject the disturbances [14]. The controller performance was compared with a PID controller. Step and square trajectories are used in the comparison. The controllers' parameters are chosen to render the closed-loop characteristic polynomial with no focus on the system robustness. An improved adaptive fuzzy backstepping control is shown in the cited reference [15]. Sine trajectory was only used to compare the performance of the proposed controller with sliding mode and command filtered adaptive backstepping controllers. The parameters of the proposed controller are tuned only. Feedback linearization based linear quadratic regulator with integral action (FL-LQR-I) was designed [16]. The controller performance was compared with LQR and PID controllers for three reference trajectories (Step, square, and sine). Salp Swarm Algorithm (SSA) were utilized to tune the controllers' parameters. Motivated by the aforementioned research, this paper presents a sliding mode controller with an integral term (SM-I). The proposed controller is compared with LQR and PID controllers using step, sine, and square trajectories. All the controllers' parameters are tuned using EO to ensure a fair comparison. The proposed (SM-I) controller can track step, sine, and square trajectories even when all the MLS parameters are changed by $30 \%$ while the change in the ref. [16] is $10 \%$ only. Moreover, it offers a better tracking response than the one in the ref. [16] in terms of overshoot and settling time.

It is arduous to manually tune or design the gains of the nonlinear controller. To overcome this issue, an optimization method can be utilized to tune the nonlinear controller gains. These are numerous types of optimization techniques and there are several factors for choosing between them. EO, developed by Afshin Faramarzi et al. [17], is a physics-based optimization method that is inspired by control volume mass balance models used to estimate the dynamic and equilibrium states. The EO provides some advantages such as the ease of implementation, the capability of offering a balance between the exploitation and exploration operators as well as the diversity among the individuals in a population.

The main contributions of this paper include: i) proposing a new application of the EO algorithm to tune the gains of the sliding mode control of the MLS, ii) introducing a novel 
performance index that evaluates both the control performance and control effort called the Integral Absolute Controller Output-weighted Error (IACOE) and iii) reducing the chattering, i.e., smoothing the system output, by letting the EO find the best slope $(\mu)$ for signum function in (13).

The remainder of this paper is structured as follows: Section 2 presents the modelling of the magnetic levitation system. Sections 3 and 4 show the design and analysis of the proposed controller. Section 5 presents the EO. Section 6 demonstrates and discusses the results of the simulation in detail. Section 7 presents the conclusions of the paper and highlights the future work to be pursued.

\section{MLS MODEL}

The MLS and its diagram used in this work are illustrated in Figure 1. The nonlinear mathematical model of MLS, which is derived based on the works [18-20], is stated below. The state equations that define the nonlinear physical model of the MLS are obtained from the principal laws of physics of a sphere motion in the electromagnetic field.
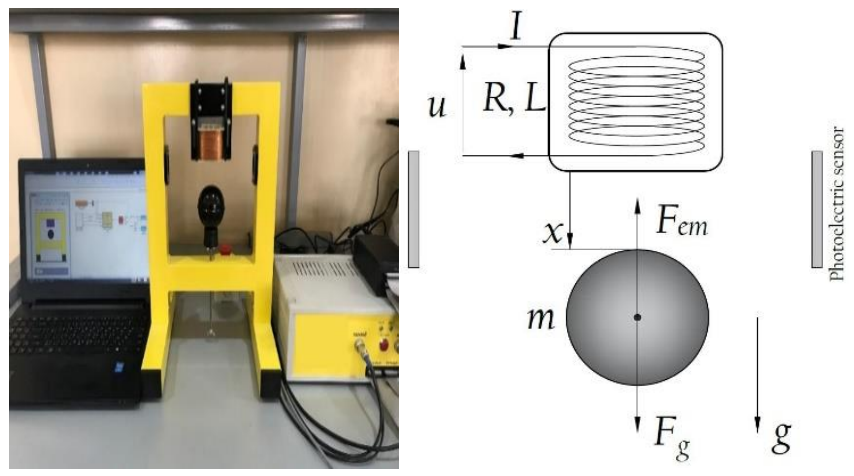

Figure 1. MLS and magnetic levitation diagram

The Lagrangian function, which represents the kinetic energy minus the potential energy, describes the electromagnetic forces and ball dynamics (see the right side of Figure 1) in the following:

$$
T=\frac{1}{2} m \dot{x}^{2}+\frac{1}{2} L(x) \dot{q}^{2}+\frac{1}{2} \int_{0}^{t} R \dot{q}^{2} d t+m g x+q u
$$

where, $m$ denotes the mass of the ball, $g$ is the gravity constant, $x$ describes the distance of the ball from the electromagnet, $L(x)$ is a function that describes the dependence of coil inductance on $x, u$ represents the voltage, $I=\dot{q}$ denotes the current in the coil, $R$ describes the resistance of the electromagnet coil, $q$ represents the electric charge, $F_{e m}$ is the electromagnetic force, and $F_{g}$ is the gravity force.

The variables $q(t)$ and $x(t)$ must fulfil the Lagrangian equations: $\frac{d}{d t} \frac{d T}{d \dot{x}}-\frac{\partial T}{\partial x}=0$ and $\frac{d}{d t} \frac{d T}{d \dot{q}}-\frac{\partial T}{\partial q}=0$ which yield Newton's second law and Kirchhoff's voltage law as follows:

$$
\begin{gathered}
\frac{d^{2} x}{d t^{2}}=\frac{1}{2 m} \frac{d L}{d x} I^{2}+g \\
\frac{d I}{d t}=\frac{1}{L}\left(-\frac{d L}{d x} \frac{d x}{d t} I-R I+u\right)
\end{gathered}
$$

The electromagnetic force is described by:

$$
F(x, I)=\frac{1}{2} \frac{d L}{d x} I^{2}
$$

The exponential function is a good approximation of $L(x)$ as follows:

$$
L(x)=L_{0}+L_{1} e^{-a x} \quad a>0
$$

where, $L_{0}$ is the inductance of the coil when the ball is very far away $(x=\infty), L_{l}$ is the incremental inductance when there is a touching between the coil and the ball ( $\mathrm{x}=0), a$ denotes the length constant, $L_{1} \approx F_{e m P 1}, \quad$ and $a \approx \frac{1}{F_{e m P 2}}$, where $F_{e m P 1}$ and $F_{e m P 2}$ are electromagnetic force parameters which are gained by measuring the dependence of coil current on ball position in steady-state. Since $L(x)$ is decreasing, its derivative is always negative. The exponential alternative of (3) will be used by having:

$$
\frac{d L}{d x}=-a L_{1} e^{-a x}
$$

where, $\frac{d L}{d x} \approx-\frac{F_{\text {emP1 }}}{F_{\text {emP2 }}} e^{-\left(\frac{x_{1}}{F_{\text {emP2 }}}\right)}$. The experimentally received approximation in [19] simplifies Eq. (2) as follows:

$$
\frac{d I}{d t}=-\frac{1}{f(x)}(k u+c-I)
$$

where, $c$ and $k$ are given by approximation of real measured data, and $\mathrm{f}(x)$ is the time constant that has an exponential alternative similar to (4). By introducing the state variables as below and combining (1), (2), (4) and (5) with slightly different notation as follows: $x_{1}=x$; is the position of the ball, $x_{2}=\dot{x}_{1}$; is the velocity of the ball, and $x_{3}=I$; is the current of the coil. The resulting nonlinear mathematical model is written as follows:

$$
\begin{gathered}
\dot{x}_{1}=x_{2} \\
\dot{x}_{2}=-\frac{F_{e m}}{2 m}+g \\
\dot{x}_{3}=\frac{1}{f\left(x_{1}\right)}\left(k u+c-x_{3}\right)
\end{gathered}
$$

where, $\quad F_{e m}=x_{3}^{2} \frac{F_{e m P 1}}{F_{e m P 2}} e^{-\left(\frac{x_{1}}{F_{e m P 2}}\right)}, \quad$ and $f\left(x_{1}\right)=$ $\frac{f_{P 1}}{f_{P 2}} e^{-\left(\frac{x_{1}}{f_{P 2}}\right)} \cdot f_{P 1}$ and $f_{P 2}$ are given by approximation of real measured data. For more details about the MLS Model, please refer to the ref. [18].

\section{SLIDING MODE CONTROL}

Starting with the following single-input single-output system, which is in the normal form:

$$
\begin{gathered}
\dot{\xi}_{i}=\xi_{i+1} \text { for } 1 \leq i \leq n-1 \\
\dot{\xi}_{n}=a(\xi)+b(\xi) u+\delta(t, \xi, u) \\
y=\xi_{1}
\end{gathered}
$$

where, $\quad \xi=\operatorname{col}\left(\xi_{1}, \ldots, \xi_{n}\right) \in D_{\xi} \subset R^{n} \quad$ for $\quad$ a domain $D_{\xi}$ and $y$ is the output. The functions a and $\mathrm{b}$ are locally Lipschitz and $b \neq 0$ over $D_{\xi}$ and $\delta$ represents a time- 
varying disturbance assumed to piecewise continuous in $\mathrm{t}$ and locally Lipschitz in $(\xi, u)$. The system (7) is obtained from (6) by using the feedback linearization technique, which is explained in detail in the ref. [16]. Suppose $r(t)$ is the desired reference signal, then the change of variables:

$$
e_{1}=\xi_{1}-r=y-r, \mathrm{e}_{2}=\xi_{2}, \ldots, \mathrm{e}_{n}=\xi_{n}
$$

transforms the system (7) into the form:

$$
\begin{aligned}
\dot{e}_{i} & =e_{i+1} \quad \text { for } 1 \leq i \leq n-1 \\
\dot{e}_{n} & =a(\xi)+b(\xi) u+\delta(t, \xi, u)
\end{aligned}
$$

The goal is to design a controller that constrains the trajectories of the system to a sliding manifold or surface. Starting with the linear part of (8) as follows:

$$
\dot{e}_{i}=e_{i+1}, 1 \leq i \leq n-1
$$

$e_{n}$ can be designed to stabilize the origin of (9) when it is viewed as the control input as follows:

$$
e_{n}=-\left(k_{1} e_{1}+\ldots+k_{n-1} e_{n-1}\right)
$$

where, $k_{1}$ to $k_{n-1}$ are selected such that the polynomial $\lambda^{n-1}+k_{n-1} \lambda^{n-2}+\ldots+k_{1}$ is Hurwitz. The sliding manifold is then designed as [21],

$$
s=\left(k_{1} e_{1}+\ldots+k_{n-1} e_{n-1}\right)+e_{n}=0
$$

and its derivative

$$
\dot{s}=\sum_{1}^{n-1} k_{i} e_{i+1}+a(\xi)+b(\xi) u+\delta(t, \xi, u)
$$

The motion on the manifold $s=0$ is independent of $a$ and $b$. The task now is to design a controller to bring the system trajectories to the aforementioned manifold and maintain it there. Let $V=\frac{s^{2}}{2}$ be a Lyapunov function candidate and the control input $u$ be chosen as:

$$
u=\frac{1}{b(\xi)}[-a(\xi)+v]
$$

Then, $\dot{V}$ becomes,

$$
\dot{V}=s\left[\sum_{1}^{n-1} k_{i} e_{i+1}+v+\delta(t, \xi, u)\right] \leq|s| \varrho(\xi)+s v
$$

where, $\quad \varrho(\xi) \geq\left|\sum_{1}^{n-1} k_{i} e_{i+1}+\delta(t, \xi, u)\right|, \forall t>0, u \in$ $R$ and $\xi \in D_{\xi}$. The selection of $v$ is done to meet the following: the term $s v$ is negative and dominates the positive term $|s| \varrho(\xi)$ when $|s| \neq 0$ and the net negative term to drive $|s|$ to zero in finite time. This can be accomplished by choosing

$$
v=-\beta(\xi) \operatorname{sgn}(s)
$$

where, $\quad \beta(\xi) \geq \varrho(\xi)+\beta_{0}, \beta_{0}>0$ and the signum function $\operatorname{sgn}(\cdot)$ is defined by:

$$
\operatorname{sgn}(s)=\left\{\begin{aligned}
1, & s>0 \\
0, & s=0 \\
-1, & s<0
\end{aligned}\right.
$$

whenever $|s| \neq 0$, then

$$
\dot{V} \leq|s| \varrho(\xi)-\left[\varrho(\xi)+\beta_{0}\right] \operatorname{sgn}(s) \leq-\beta_{0}|s| \leq-\beta_{0} \sqrt{2 V}
$$

The inequality $\dot{V} \leq-\beta_{0} \sqrt{2 V}$ guarantees that all the trajectories starting away from the manifold $s=0$ reach it in a finite time and those on the manifold cannot leave it. The control law (11) is discontinuous and oscillates with very high (ideally infinite) frequency. This cannot be realized practically due to limited actuator bandwidth. This yields deviation of the trajectory of the sliding manifold causing it to oscillate around it in what is called chattering. To avoid the infinite-frequency oscillation in the control law (11), the signum function is replaced by a high slope saturation function as follows:

$$
v=-\beta(\xi) \operatorname{sat}\left(\frac{S}{\mu}\right)
$$

where, sat $(\cdot)$ denotes the saturation function defined by:

$$
\operatorname{sat}\left(\frac{s}{\mu}\right)=\left\{\begin{array}{cc}
\frac{s}{\mu} & \text { if }\left|\frac{s}{\mu}\right| \leq 1 \\
\operatorname{sgn}\left(\frac{s}{\mu}\right) & \text { if }\left|\frac{s}{\mu}\right|>1
\end{array}\right.
$$

as $\mu \rightarrow 0$, sat $\left(\frac{s}{\mu}\right)$ approaches $\operatorname{sgn}(s)$. The control law (12) is called SM and $\beta(\xi)=k_{1}\left|\zeta_{1}\right|+k_{2}\left|\zeta_{2}\right|+\beta_{0}$ for the system (6), i.e., $n=3$. The parameters $k_{1}, k_{2}, \beta_{0}$, and $\mu$ are tuned by EO. The derivative $\dot{V}$ of the Lyapunov function $V=\frac{s^{2}}{2}$ while using the control law (12) is given by $\dot{V}=-\beta_{0}|s|$ when $|s| \geq \mu$, i.e., outside the boundary layer $\{|s| \leq \mu\}$, Hence, whenever $|s(0)|>\mu,|s(t)|$ is strictly decreasing until it reaches the set $\{|s| \leq \mu\}$ in a finite time and remains inside it thereafter.

To study the behaviour of $e(t)$, let $\zeta=\operatorname{col}\left(e_{1}, \ldots, e_{n-1}\right)$, which satisfies the equation

$$
\dot{\zeta}=\left(A_{c}-B_{c} K\right) \zeta+B_{c} S
$$

where, $K=\left[k_{1}, k_{2}, \ldots, k_{n-1}\right], A_{c}=\left[\begin{array}{ll}0 & 1 \\ 0 & 0\end{array}\right]_{n=3}$, and $B_{c}=$ $\left[\begin{array}{l}0 \\ 1\end{array}\right]_{n=3}$. The matrix $\left(A_{c}-B_{c} K\right)$ is Hurwitz by the design of (10). Let the Lyapunov function candidate $V_{0}=$ $\zeta^{T} P \zeta$ where $P$ the solution of the Lyapunov equation $P\left(A_{c}-\right.$ $\left.B_{c} K\right)+\left(A_{c}-B_{c} K\right)^{T} P=-I$. The derivative of $V_{0}$ satisfies the inequality,

$$
\begin{gathered}
\dot{V}_{0}=-\zeta^{T} \zeta+2 \xi^{T} P B_{c} s-\leq(1-\theta)\|\zeta\|^{2}, \\
\forall\|\zeta\| \geq 2\left\|P B_{c}\right\||s| / \theta
\end{gathered}
$$

where, $0<\theta<1$. Using Rayleigh-Ritz theorem, $\zeta^{T} P \zeta \leq$ $\lambda_{\max }(P)\|\zeta\|^{2}$. For any $\sigma \geq \mu,\left\{\|\zeta\| \leq 2\left\|P B_{c}\right\| \sigma / \theta\right\} \subset$ $\left\{\zeta^{T} P \xi \leq \lambda_{\max }(P)\left(2\left\|P B_{c}\right\| / \theta\right)^{2} \sigma^{2}\right\} \quad$. Choosing $\rho_{1}=$ $\lambda_{\text {max }}(P)\left(2\left\|P B_{c}\right\| / \theta\right)^{2}$ and $c$ such that $c>\mu$ yields the set $\Omega=\left\{\zeta^{T} P \xi \leq \rho_{1} c^{2}\right\} \times\{|s| \leq c\}$, which is positively invariant since $\dot{V}<0$ on the boundary $\{|s|=c\}$ and $\dot{V}_{0}<$ 0 on the boundary $\left\{\zeta^{T} P \xi=\rho_{1} c^{2}\right\}$. if $e(0) \in \Omega$, 
then $e(t)$ enters the set $\Omega_{\mu}=\left\{\zeta^{T} P \xi \leq \rho_{1} \mu^{2}\right\} \times\{|s| \leq \mu\}$ in finite time because it is positively invariant. Inside $\Omega_{\mu}$ to calculate the ultimate bound on $\left|e_{1}\right|$, the following equation [21] is utilized:

$$
\max _{x^{T} P x \leq c}\left|b^{T} x\right|=\sqrt{c}\left\|b^{T} P^{\frac{-1}{2}}\right\|
$$

Thus, $\max _{\zeta^{T} \zeta \leq \rho_{1} \mu^{2}}\left|e_{1}\right|=\sqrt{\rho_{1} \mu^{2}}\left\|b^{T} P^{\frac{-1}{2}}\right\| \quad$ where $\quad b^{T}=$ $[1,0, \ldots, 0]$. Setting $\hat{k}=\left\|b^{T} P^{\frac{-1}{2}}\right\| \sqrt{\rho_{1}}$ shows that the ultimate bound on $\left|e_{1}\right|$ is $\hat{k} \mu$. Ultimate boundedness can be concluded as the disturbance $\delta$ could be nonvanishing at the origin.

\section{SLIDING MODE CONTROL WITH INTEGRAL ACTION}

The sliding mode controller of section 3 assures that the regulation error $y-r$ is ultimately bounded by $\hat{k} \mu$ with $\hat{k}>0$. Using the integral action, it can be assured that the error converges to zero as time tends to infinity. Augmenting the integrator $\dot{e}_{0}=y-r$ with (8) yields

$$
\begin{gathered}
\dot{e}_{i}=e_{i+1} \text { for } 0 \leq i \leq n-1 \\
\dot{e}_{n}=a(\xi)+b(\xi) u+\delta(t, \xi, u)
\end{gathered}
$$

which preserves the normal form structure with a chain of $n+$ 1 integrators. The design of sliding mode can be performed in a similar way to section 3 . The sliding manifold is taken as [21].

$$
s=\left(k_{0} e_{0}+k_{1} e_{1}+\ldots+k_{n-1} e_{n-1}\right)+e_{n}=0
$$

where, $k_{0}$ to $k_{n-1}$ are chosen such that the polynomial $\lambda^{n}+$ $k_{n-1} \lambda^{n-1}+\ldots+k_{1} \lambda_{1}+k_{0}$ is Hurwitz. The control law u can be designed as:

$$
u=\frac{1}{b(\xi)}\left[-a(\xi)-\beta(\xi) \operatorname{sat}\left(\frac{s}{\mu}\right)\right]
$$

where, $\quad \beta(\xi) \geq \varrho(\xi)+\beta_{0}, \beta_{0}>0, \varrho(\xi) \geq \mid \sum_{0}^{n-1} k_{i} e_{i+1}+$ $\delta(t, \xi, u) \mid \forall t>0, u \in R$ and $\xi \in D_{\xi}$; and $\operatorname{sat}\left(\frac{s}{\mu}\right)$ is as in (13). The control law (14) is called SM-I and $\beta(\xi)=k_{0}\left|\zeta_{0}\right|+$ $k_{1}\left|\zeta_{1}\right|+k_{2}\left|\zeta_{2}\right|+\beta_{0}$ for the system (6), i.e., $n=3$. The parameters $k_{0}, k_{1}, k_{2}, \beta_{0}$, and $\mu$ are tuned by EO. The behaviour of $e(t)$ is studied similarly as in section 3. It can be shown that there are two compact positively invariant sets $\Omega=$ $\left\{\zeta^{T} P \xi \leq \rho_{1} c^{2}\right\} \times\{|s| \leq c\} \quad$ and $\quad \Omega_{\mu}=\left\{\zeta^{T} P \xi \leq \rho_{1} \mu^{2}\right\} \times$ $\{|s| \leq \mu\}$ for $c>\mu$ such that every trajectory starts in $\Omega$ enters $\Omega_{\mu}$ in a finite time. Inside $\Omega_{\mu}$, the system possesses an equilibrium at $(\zeta=\bar{\zeta}, s=\bar{s})$ where $\bar{\zeta}=$ $\operatorname{col}\left(\bar{e}_{0}, 0, \ldots, 0\right)$ and $\bar{s}=k_{0} \bar{e}_{0}$. The change of variables $v=$ $\zeta-\bar{\zeta}$ and $\tau=s-\bar{s}$ is used to shift the equilibrium point to the origin. Then, the closed-loop system is given by:

$$
\begin{gathered}
\dot{v}=\left(A_{c}-B_{c} K\right) v+B_{c} \tau \\
\dot{\tau}=-\beta(\xi) \frac{\tau}{\mu}+\left[\sum_{1}^{n-1} k_{i} e_{i+1}+\delta(t, v, u)\right]
\end{gathered}
$$

where, $\delta(t, 0, u)=0$. Composite Lyapunov function can be constructed as $V=v^{T} P v+\frac{\tau^{2}}{2}$ whose derivative satisfies,

$$
\begin{aligned}
\dot{V} & =2 v^{T} P\left[\left(A_{c}-B_{c} K\right) v+B_{c} \tau\right]-\beta(\xi) \frac{\tau^{2}}{\mu}+ \\
\tau & {\left[\sum_{1}^{n-1} k_{i} e_{i+1}+\delta(t, \xi, u)\right] } \\
& \leq v^{T}\left[\left(A_{c}-B_{c} K\right)^{T} P+P\left(A_{c}-B_{c} K\right)\right] v+ \\
& 2\left\|P B_{c}\right\|\|v\||\tau|-\frac{\beta(\xi)}{\mu}|\tau|^{2}+|\varrho(\xi) \| \tau|
\end{aligned}
$$

since $\varrho(\xi)$ is locally Lipschitz and smooth, it can be upper bounded by:

$$
|\varrho(\xi)| \leq \hat{k}_{1}\|v\|+\hat{k}_{2}|\tau|
$$

where, $\hat{k}_{1}>0$ and $\hat{k}_{2}>0$. Thus, $\dot{V}$ becomes

$$
\begin{gathered}
\dot{V} \leq-\|v\|^{2}+2\left(\left\|P B_{c}\right\|+\frac{\hat{k}_{1}}{2}\right)\|v\||\tau|-\left(\frac{\beta(\xi)}{\mu}-\hat{k}_{2}\right)|\tau|^{2} \\
\leq-\left[\begin{array}{c}
\|v\| \\
\tau
\end{array}\right]^{T}\left[\begin{array}{cc}
1 & -\left(\left\|P B_{c}\right\|+\frac{\hat{k}_{1}}{2}\right) \\
-\left(\left\|P B_{c}\right\|+\frac{\hat{k}_{1}}{2}\right) & \frac{\beta(\xi)}{\mu}-\hat{k}_{2}
\end{array}\right]\left[\begin{array}{c}
\|v\| \\
\tau
\end{array}\right]
\end{gathered}
$$

The right-hand side of $\dot{V}$ is negative-definite for $\mu<$ $\frac{\hat{\beta}_{0}}{\left[\hat{k}_{2}+\left(\left\|P B_{C}+\frac{\widehat{k}_{1}}{2}\right\|\right)^{2}\right]}$ where $\beta(\xi)<\hat{\beta}_{0}$. Hence, every trajectory in $\Omega_{\mu}$ converges to the equilibrium point $(v=0, \tau=$ 0 ) as $t \rightarrow 0$. Since $e=0$ at this point, it can be concluded that the error converges to zero, i.e., $y \rightarrow r$.

\section{EQUILIBRIUM OPTIMIZER (EO)}

EO is a meta-heuristic algorithm, recently proposed by Afshin Faramarzi et al. [17], which is based on a simple wellmixed dynamic mass balance on a control volume. More details about the EO inspiration is stated in the ref. [17]. The following steps show the mathematical model of the EO algorithm:

\section{Step 1: Initialization}

In this step, EO uses the initial population to start the process of optimization. The vector of initial concentrations is constructed randomly in the search space as follows:

$$
\begin{aligned}
C_{i}^{\text {initial }}= & C_{\min }+\operatorname{rand}_{i}\left(C_{\max }-C_{\min }\right) \\
& i=0,1,2, \ldots \ldots, n
\end{aligned}
$$

where, $C_{i}^{\text {initial }}$ denotes the initial concentration vector of a particle $i$, rand $_{i}$ specifies a random vector in the range of [0, 1], $C_{\text {max }}, C_{\text {min }}$ are the maximum and minimum and values for each dimension in the problem, and $n$ denotes the number of particles in the population.

\section{Step 2: Equilibrium pool and candidates $\left(C_{\text {eq,pool }}\right)$}

EO seeks for the equilibrium state of the system, which is desired to be the global optimum and close to the best solution. A vector named the equilibrium pool is constructed from five candidates, which support having better diversification and 
exploitation capabilities as follows:

$$
\vec{C}_{e q, p o o l}=\left\{\vec{C}_{e q(1),} \vec{C}_{e q(2),} \vec{C}_{e q(3),} \vec{C}_{e q(4),} \vec{C}_{e q(\text { ave })}\right\}
$$

\section{Step 3: Updating the concentration}

The next term (F) supports EO in having a plausible balance between exploitation and exploration.

$$
\vec{F}=e^{\left(\left[-\vec{\lambda}\left(t-t_{0}\right)\right]\right)}
$$

where, $\vec{\lambda}$ is assumed to be a vector with random numbers in the range of $[0,1], \mathrm{t}$ is a variable that retains a reverse proportionality with the iteration numbers, and $t_{0}$ is a vector that supports to guarantee the convergence by improving the exploitation and exploration capability of the algorithm while slowing down the search speed. Finally, the updating rule of EO is as follows:

$$
\vec{C}=\vec{C}_{e q}+\left(\vec{C}_{0}-\vec{C}_{e q}\right) \cdot \vec{F}+\underset{\overrightarrow{\lambda V}}{\frac{\vec{G}}{\vec{\lambda}}}(1-\vec{F})
$$

where, $V$ is considered as a unit, $\vec{G}$ is the generation rate, which is used to improve the exploitation phase. For more details about the EO and its pseudo-code please refer to the cited ref. [17].

\section{RESULTS AND DISCUSSION}

In this section, simulation studies by using the MATLAB software are performed to demonstrate the effectiveness of the derived nonlinear controller (SM-I). The MLS parameters are as follows: $m=0.02855 \mathrm{~kg}, f_{p_{1}}=1.4142 *$ $10^{-4} \mathrm{~m} . \mathrm{s}, f_{p_{2}}=4.5626 * 10^{-3} \mathrm{~m}, f_{e m P 1}=1.7521 *$ $10^{-2} \mathrm{H}, f_{\text {emP2 }}=5.8231 * 10^{-3} \mathrm{~m}, k=2.5165 \mathrm{~A} / \mathrm{V}, \mathrm{c}=$ $0.0243 A$, and $g=9.8100 \mathrm{~m} / \mathrm{s}^{2}$. The MLS used has the following limitations: $\quad x_{1 M I N}=0 \mathrm{~m} \quad, \quad x_{1 M A X}=$ $0.0200 \mathrm{~m}, x_{3 M I N}=0.0388 \mathrm{~A}, x_{3 M A X}=2.345 \mathrm{~A}, U_{M I N}=$ $0.0 \mathrm{~V}$, and $U_{M A X}=5.0 \mathrm{~V}$. The initial conditions of the MLS states are $x(0)=[0.004 \mathrm{~m}, 0 \mathrm{~m} / \mathrm{s}, 0.608 \mathrm{~A}]^{T}$. The sampling frequency is chosen as $f=1 \mathrm{kHz}$.

Two simulation scenarios are considered to validate the theoretical background and the possible advantage of the proposed control method. The first scenario considers taking the MLS without any perturbation in its parameters to study the proposed controller under the calculated MLS parameters. The second scenario demonstrates the capability of the proposed control to handle the uncertainty in MLS parameters which is a good measure of the robustness of the proposed controller. In both scenarios, three reference trajectories are tested and comparative simulation studies are investigated. These reference trajectories are Step, Sinusoidal, and Square, which are represented in the following equations: $x_{r}(t)=$ $0.009 u(t)$ for Step, $x_{r}(t)=0.0025 \sin (0.5 \pi t)+0.009$ for Sinusoidal, and $\quad x_{r}(t)=0.0025 \operatorname{sign}(\sin (0.5 \pi t))+$ 0.009 for Square.

In these comparative simulation studies, the proposed controller (SM-I) is compared with SM, LQR, and PID controllers. Because they are widely used in controlling the MLS, the LQR and PID controllers are selected for the comparison. The purpose of comparing the SM-I and SM controllers is to demonstrate the enhancement in the robustness of the system in the presence of the integral term.

To ensure a fair comparison, the gains of the SM-I, SM, LQR, and PID controllers are tuned using EO algorithm under analogous conditions. The EO is run many times for each controller to obtain the minimum cost function with different lower and upper bounds. It is essential to select the correct cost functions or performance indexes to obtain the best performance of the system and to fulfil the design expectations for the controller. The performance index used in this work is the Integral Time-weighted Square Error (ITSE) since it provides shorter settling and rise times due to its large controller output, namely a faster response of the system. The error used in ITSE is the error in the ball position. ITSE is calculated as $J=\int_{t_{1}}^{t_{2}} t \cdot e^{2} d t$ where $e$ is as defined above, and $\mathrm{t}$ is the period $\left(t_{1}, t_{2} \in 0,1.5\right.$ for step reference trajectory and $t_{1}, t_{2} \in 0,14$ for square and sine reference trajectories).

The EO algorithm parameters such as the maximum iteration and the number of search agents affect the speed of the algorithm to find the optimal solution and the possibility to find the global optimal. The selection of these parameters value is application related. MLS is a nonlinear system with a high possibility of multi-local optimum which makes the selection of suitable EO parameters is more difficult. Since, the proposed work is a new application of the recently introduced EO algorithm, the selection of the EO parameters is based on trial and error, and personal experience. Accordingly, the EO parameters are chosen as 150 and 15 for the maximum iteration and the number of search agents respectively. Table 1 demonstrates the upper and lower bounds for the tuned parameters while Table 2 shows best-obtained values of the tuned gains. $K_{3}=\beta_{0}$ in Table 1 and Table 2 .

Table 1. The bounds of the tuned gains

\begin{tabular}{cccccc}
\hline \multirow{3}{*}{ Controller } & \multicolumn{5}{c}{ Lower bounds, Upper bounds } \\
& $\mathbf{K}_{\mathbf{1}}, \mathbf{Q}_{\mathbf{1}, \mathbf{1}}$ & $\mathbf{K}_{\mathbf{2}}, \mathbf{Q}_{\mathbf{2}, \mathbf{2}}$ & $\mathbf{K}_{\mathbf{3}}, \mathbf{Q}_{\mathbf{3}, \mathbf{3}}$ & $\boldsymbol{\mu}, \mathbf{R}$ & $\mathbf{K}_{\mathbf{0}}$ \\
& $\mathbf{K}_{\mathbf{p}}$ & $\mathbf{K}_{\mathbf{I}}$ & $\mathbf{K}_{\mathbf{D}}$ & & \\
\hline SM+I & $0,10^{4}$ & $0,10^{3}$ & $0,10^{3}$ & $10^{-3}, 1$ & $5 * 10^{3}, 10^{4}$ \\
SM & $0,10^{4}$ & $0,10^{3}$ & $0,2 * 10^{3}$ & $10^{-3}, 1$ & --- \\
PID & $0,10^{3}$ & $0,10^{3}$ & $0,10^{3}$ & -- & --- \\
LQR & $1,10^{4}$ & $1,10^{4}$ & 1,50 & 1,20 & --- \\
\hline
\end{tabular}

Table 2. The tuned values of the controllers' gains

\begin{tabular}{cccccc}
\hline \multirow{2}{*}{ Controller } & \multicolumn{5}{c}{ Parameters } \\
& $\mathbf{K}_{\mathbf{1}, \mathbf{P}}$ & $\mathbf{K}_{\mathbf{2}, \mathbf{I}}$ & $\mathbf{K}_{\mathbf{3}, \mathbf{D}}$ & $\boldsymbol{\mu}$ & $\mathbf{K}_{\mathbf{0}}$ \\
\hline SM+I & 5000 & 142.6 & 1900 & 0.903 & $3 \times 10^{3}$ \\
SM & 5500 & 130.3 & 1986 & 0.988 & --- \\
PID & 256.1 & 1000 & 6.06 & --- & --- \\
LQR & 168.7 & 3.82 & 0.548 & --- & --- \\
\hline
\end{tabular}

\subsection{Reference trajectory tracking test (without perturbation)}

In this test, the controllers' reference trajectory tracking performance is evaluated with the aforementioned reference trajectories applied to the MLS that possesses no perturbation in its parameters. The LQR and SM controllers provide a shorter settling time a smaller overshoot than the SM-I and PID controllers as shown in Figure 2. However, in the same Figure, the SM-I and PID controllers offer a shorter rise time of $50.4 \mathrm{~ms}$ and $38.6 \mathrm{~ms}$ respectively, which is desirable in the MLS application, compared with the SM and LQR controllers 
that offer a longer rise time of $54.3 \mathrm{~ms}$ and $68.7 \mathrm{~ms}$ respectively. The rise time is calculated for the interval [0\% 90\%] of the steady-state value. As observed in Figures 3 and 4, the deviation between the desired and measured ball position by using the LQR controller is very large as compared to the other controllers.

The SM-I controller in Figure 3 shows a better tracking performance to the Sinusoidal trajectory than the PID, LQR, and SM controllers, particularly in the growing and dropping intervals. As noticed in Figure 4, the SM-I the controller has successfully followed the Square trajectory with an acceptable deviation in the falling and rising stages.

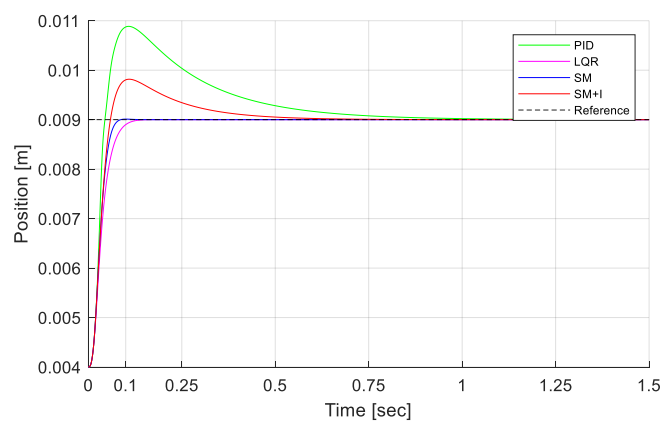

Figure 2. MLS response for step trajectory without parameter perturbations

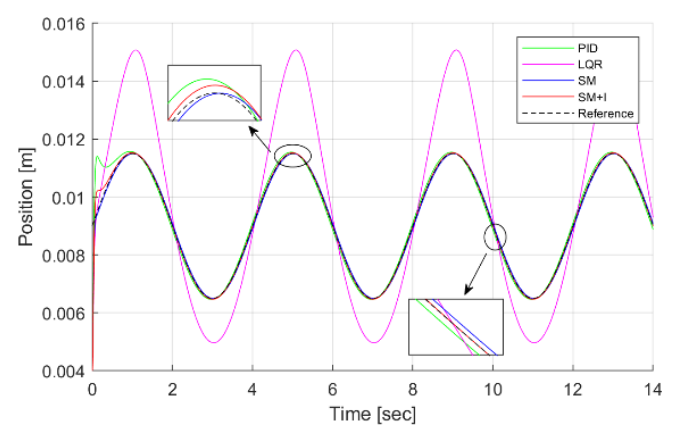

Figure 3. MLS response for sine trajectory without parameter perturbations

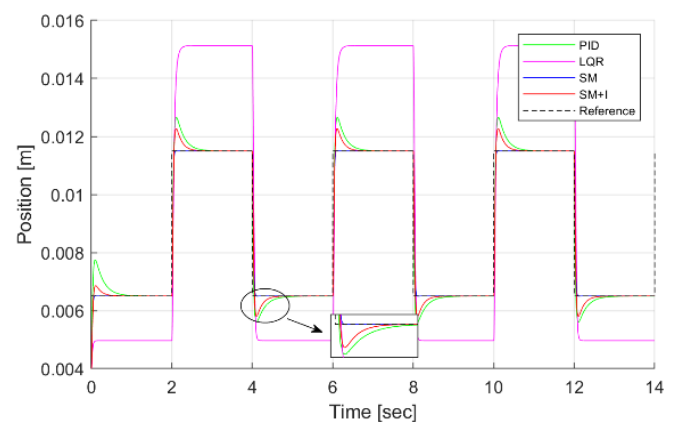

Figure 4. MLS response for square trajectory without parameter perturbations

\subsection{Robustness test}

The three references trajectories, in this test, are fed to the MLS, which retains an uncertainty in its parameters. The values of $k, c, f_{P_{1}}, f_{P_{2}}, f_{e m P 1}$, and $f_{e m P 2}$, are changed by $+30 \%$. The controllers' performance is evaluated under this perturbation.
As can be observed in Figures 5-7, the SM and LQR controllers are not able to track the reference trajectories without an SSE, which is significantly large in the case of the LQR controller. On the other hand, The PID controller provides a better tracking performance for the three reference trajectories than the SM and LQR controllers; however, the settling time and overshoot are still unacceptable. The SM-I controller offers a superior tracking performance for the three reference trajectories in both steady and transient response. The SM-I controller has shown robustness to parameters change, which can be seen by comparing it with the other controllers. Additionally, it can be observed from Figure 7 that there is a variance in the control performance between the falling and rising stages. The main reason for this difference may be the electromagnet retains nonlinearity and asymmetrically in its magnetic field.

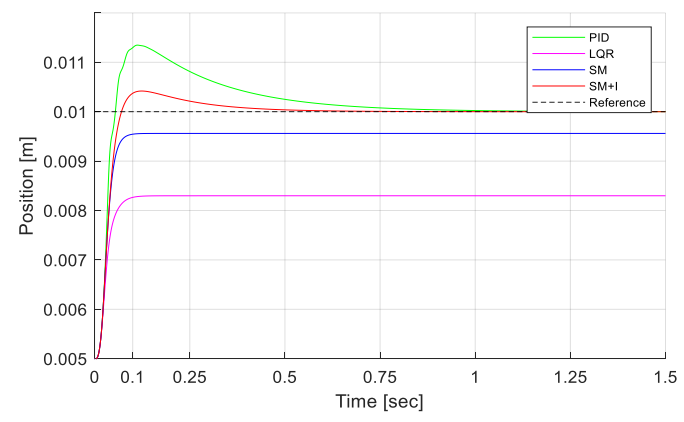

Figure 5. MLS response for step trajectory with parameter perturbations

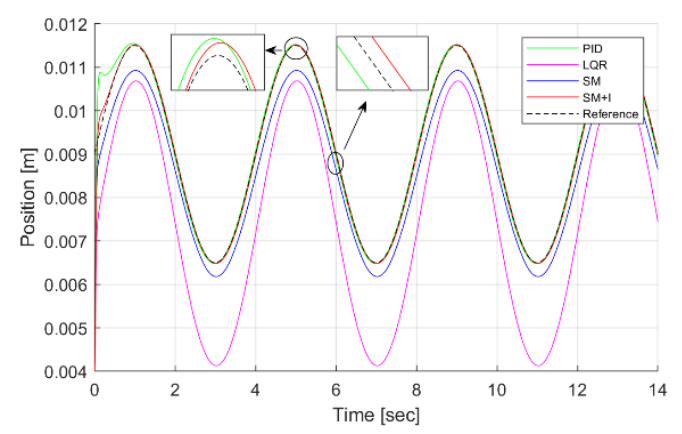

Figure 6. MLS response for sine trajectory with parameter perturbations

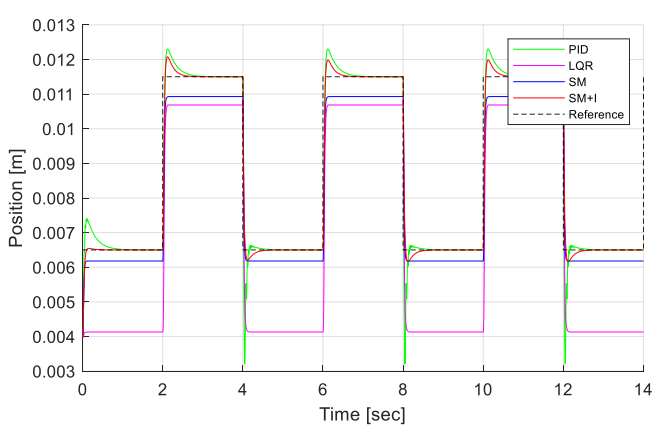

Figure 7. MLS response for square trajectory with parameter perturbations

The comparison of the controllers is also numerically supported as shown in Table 3 and 4. These numerical values are created from the evaluation of the SM-I, SM, LQR, and 
PID controllers' effort and performance with two test functions.

Table 3. IAE with perturbation

\begin{tabular}{cccc}
\hline \multirow{2}{*}{ Controller } & \multicolumn{3}{c}{$\int_{\boldsymbol{t}_{\mathbf{1}}}^{\boldsymbol{t}_{2}}|\boldsymbol{e}(\boldsymbol{t})| \boldsymbol{d t}$} \\
& Step & Sine & Square \\
\hline SM & $97.03 \times 10^{-5}$ & $644.8 \times 10^{-5}$ & $67.11 \times 10^{-4}$ \\
SM+I & $\mathbf{2 6 . 3 6 \times 1 0 ^ { - 5 }}$ & $\mathbf{6 7 . 4 9 \times 1 0 ^ { - 5 }}$ & $\mathbf{1 4 . 5 \times 1 0 ^ { - 4 }}$ \\
PID & $53.69 \times 10^{-5}$ & $106 \times 10^{-5}$ & $21.97 \times 10^{-4}$ \\
LQR & $266.3 \times 10^{-5}$ & $2219 \times 10^{-5}$ & $244.8 \times 10^{-4}$ \\
\hline
\end{tabular}

Table 4. IACOE with perturbation

\begin{tabular}{cccc}
\hline \multirow{3}{*}{ Controller } & \multicolumn{3}{c}{$\int_{\boldsymbol{t}_{\mathbf{1}}}^{\boldsymbol{t}_{2}}|\boldsymbol{e}(\boldsymbol{t}) \boldsymbol{u}(\boldsymbol{t})| \boldsymbol{d t}$} \\
& Step & Sine & Square \\
\hline SM & $17.12 \times 10^{-5}$ & $151.8 \times 10^{-5}$ & $154.7 \times 10^{-5}$ \\
SM+I & $\mathbf{4 . 6 8 3 \times 1 0 ^ { - 5 }}$ & $\mathbf{1 4 . 7 9 \times 1 0 ^ { - 5 }}$ & $\mathbf{3 3 . 8 6 \times 1 0 ^ { - 5 }}$ \\
PID & $10.58 \times 10^{-5}$ & $23.57 \times 10^{-5}$ & $45.53 \times 10^{-5}$ \\
LQR & $53.44 \times 10^{-5}$ & $443 \times 10^{-5}$ & $447.60 \times 10^{-5}$ \\
\hline
\end{tabular}

The first function takes the area under the curve of the error, i.e., the absolute numerical difference between the desired and measured ball position, into consideration which is also called the Integral Absolute Error (IAE) and is written as follows:

$$
\int_{t_{1}}^{t_{2}}|e(t)| d t
$$

The numerical values of the IAE of the SM-I, SM, LQR, and PID controllers for the sinusoidal and square reference trajectories are presented in Table 3. As seen in Table 3, the SM-I controller provides the smallest ball position IAE compared with the other controllers. This indicated that the SM-I controller follows the three reference trajectories with the smallest divergence compared with the other controllers.

As a measurement for control effort, many researchers have introduced performance indices such as the Integral absolute controller output (IACO) and the peak value of the controller output. When these performance indices are utilized in this work, it is noticed that the LQR controller offers the smallest control effort although it fails to track the reference trajectories without a large steady-state error. Therefore, it may be unreasonable to say that LQR offers the best control effort. Consequently, it is required to introduce a performance index that is not only constructed from the controller output.

The Integral Absolute Controller Output-weighted Error (IACOE) is a newly introduced performance index in this paper to evaluate both the control performance and control effort. IACOE (second test function) considers taking the area under the curve produced from the instantaneous multiplication of the controller output and the error resulting from the absolute difference between the reference trajectory and actual ball position as follows:

$$
\int_{t_{1}}^{t_{2}}|e(t) u(t)| d t
$$

Table 4 presents the numerical values of the IACOE of the SM-I, SM, LQR, and PID controllers for the sinusoidal and square reference trajectories. The results in Table 4 provide the superiority of the SM-I controller over the SM, LQR, and PID controllers. LQR shows the worst performance. The SM controller is not performing well which shows the benefit of adding an integral part to construct the proposed controller. The PID controller has successfully followed the three reference trajectories with an acceptable controller effort but it needs to be more improved.

\section{CONCLUSIONS}

The design of a robust sliding mode controller with an integral term to control the MLS subjected to parameter uncertainties was presented in this paper. The proposed controller's stability was guaranteed by using the Lyapunov method. The parameters of the MLS were tuned using EO to minimize the ITSE cost function, which led to minimize the tracking error and improve the robustness of the system. Simulation results verified the effectiveness of the proposed control scheme. The SM-I controller was compared with the SM, LQR, and PID controllers for three reference trajectories tracking of the MLS with and without a perturbation in the MLS parameters. The SM-I controller outperformed the other controllers despite the $30 \%$ change in the MLS parameters. As future work, the real MLS device, presented in Figure 1, will be used to validate the performance of the proposed controller.

\section{REFERENCES}

[1] Rote, D.M., Cai, Y. (2002). Review of dynamic stability of repulsive-force maglev suspension systems. IEEE Transactions on Magnetics, 38(2): 1383-1390. https://doi.org/10.1109/20.996030

[2] Peng, C., Fang, J., Xu, S. (2015). Composite antidisturbance controller for magnetically suspended control moment gyro subject to mismatched disturbances. Nonlinear Dynamics, 79(2): 1563-1573. https://doi.org/10.1007/s11071-014-1760-z

[3] van West, E., Yamamoto, A., Higuchi, T. (2009). Automatic object release in magnetic and electrostatic levitation systems. Precision Engineering, 33(3): 217228. https://doi.org/10.1016/j.precisioneng.2008.07.002

[4] Eroğlu, Y., Ablay, G. (2016). Cascade sliding modebased robust tracking control of a magnetic levitation system. Proceedings of the Institution of Mechanical Engineers, Part I: Journal of Systems and Control Engineering, 230(8): 851-860. https://doi.org/10.1177/0959651816656749

[5] Ono, M., Koga, S., Ohtsuki, H. (2002). Japan's superconducting Maglev train. IEEE Instrumentation \& Measurement Magazine, 5(1): $\quad$ 9-15. https://doi.org/10.1109/5289.988732

[6] Al-Muthairi, N.F., Zribi, M. (2004). Sliding mode control of a magnetic levitation system. Mathematical Problems https://doi.org/10.1155/S1024123X04310033

[7] Pradhan, S.K., Subudhi, B. (2016). Nonlinear control of a magnetic levitation system using a new input-output feedback linearization. IFAC-PapersOnLine, 49(1): 332336. https://doi.org/10.1016/j.ifacol.2016.03.075

[8] Bidikli, B., Bayrak, A. (2018). A self-tuning robust fullstate feedback control design for the magnetic levitation system. Control Engineering Practice, 78: 175-185. 
https://doi.org/10.1016/j.conengprac.2018.06.017

[9] Yaseen, M.H., Abd, H.J. (2018). Modeling and control for a magnetic levitation system based on SIMLAB platform in real time. Results in Physics, 8: 153-159. https://doi.org/10.1016/j.rinp.2017.11.026

[10] Zhang, X., Mehrtash, M., Khamesee, M.B. (2015). Dualaxial motion control of a magnetic levitation system using Hall-effect sensors. IEEE/ASME Transactions on Mechatronics, 21(2): 1129-1139. https://doi.org/10.1109/TMECH.2015.2479404

[11] Chopade, A.S., Khubalkar, S.W., Junghare, A.S., Aware, M.V., Das, S. (2016). Design and implementation of digital fractional order PID controller using optimal polezero approximation method for magnetic levitation system. IEEE/CAA Journal of Automatica Sinica, 5(5): 977-989. https://doi.org/10.1109/JAS.2016.7510181

[12] Pandey, S., Dourla, V., Dwivedi, P., Junghare, A. (2019). Introduction and realization of four fractional-order sliding mode controllers for nonlinear open-loop unstable system: A magnetic levitation study case. Nonlinear Dynamics, 98(1): 601-621. https://doi.org/10.1007/s11071-019-05216-x

[13] de Jesús Rubio, J., Zhang, L., Lughofer, E., Cruz, P., Alsaedi, A., Hayat, T. (2017). Modeling and control with neural networks for a magnetic levitation system. Neurocomputing, 227: 113-121. https://doi.org/10.1016/j.neucom.2016.09.101

[14] Sathiyavathi, S., Vimala Starbino, A. (2019). Design of sliding mode controller for magnetic levitation system. Computers \& Electrical Engineering, 78: 184-203. https://doi.org/10.1016/j.compeleceng.2019.07.007
[15] Sadek, U., Sarjaš, A., Chowdhury, A., Svečko, R. (2019). Improved adaptive fuzzy backstepping control of a magnetic levitation system based on Symbiotic Organism Search. Applied Soft Computing, 56: 19-33. https://doi.org/10.1016/j.asoc.2017.02.032

[16] Ismael, O.Y., Qasim, M., Noaman, M.N., Kurniawan, A. (2020). Salp swarm algorithm-based nonlinear robust control of magnetic levitation system using feedback linearization approach. In Proceedings of the 3rd International Conference on Electronics, Communications and Control Engineering, Bali, Indonesia, pp. 58-64. https://doi.org/10.1145/3396730.3396734

[17] Faramarzi, A., Heidarinejad, M., Stephens, B., Mirjalili, S. (2020). Equilibrium optimizer: A novel optimization algorithm. Knowledge-Based Systems, 191: 105190. https://doi.org/10.1016/j.knosys.2019.105190

[18] Balko, P., Rosinová, D. (2017). Modeling of magnetic levitation system. In: 2017 21st International Conference on Process Control (PC), IEEE, Strbske Pleso, Slovakia, pp. 252-257. https://doi.org/10.1109/PC.2017.7976222

[19] Bania, P. (1999). Model i sterowanie magnetyczną lewitacją. AGH, Wydział EAIiE, Katedra Automatyki, Kraków.

[20] Turnau, A., Kolek, K. (1998). Time-optimal and PID variable structure controller. In: Proceedings of the Mediterranean Conference on Electronics and Automatic Control MCEA, Morocco, pp. 476-479.

[21] Khalil, H.K. (2014). Nonlinear Control. Pearson Higher Ed. 